\title{
VENTILATORY FUNCTION TESTS. II. FACTORS AFFECTING THE VOLUNTARY VENTILATION CAPACITY
}

\author{
By HARL W. MATHESON, SIDNEY N. SPIES, JOHN S. GRAY, AND \\ DAVID R. BARNUM
}

\begin{abstract}
(From the Department of Physiology, Northwestern University Medical School, Chicago)
\end{abstract}
(Submitted for publication December 3, 1949; accepted, February 13, 1950)

The voluntary ventilation capacity is a quantitative measure of the maximum rate at which the respiratory system can carry on one of its specific and essential functions, namely, the production of an alternating flow of air in and out of the lungs. In a previous report a simple and reliable procedure for determining the voluntary ventilation capacity in man was standardized and normal values established (1). As a rational measure of the functional capacity of the pulmonary bellows, the test should be useful in the diagnosis and early detection of respiratory diseases and in evaluating the degree of functional damage. It would be of material advantage in the clinical analysis of respiratory disease, however, if in addition to measuring the degree of reduction in ventilation capacity, the causes of that impairment could likewise be identified.

In the case of a simple mechanical bellows, its functional capacity is determined by three general factors: $a$ ) its size, $b$ ) the forces operating it, and c) the resistance it offers. In the case of the pulmonary bellows, the same three factors must be concerned. The size of the pulmonary bellows can easily be measured as the vital capacity. The muscular forces available for operating the pulmonary bellows may be measured as the maximum pressure that can be developed on forced expiration. The resistance factor, which includes both the resistance to air flow in the respiratory passages and the resistance of the lung and thoracic tissues to rhythmic deformation, is not so readily measured.

It was the purpose of the present investigation to determine the effects on ventilation capacity of the vital capacity, the maximum expiratory pressure, and air-flow resistance, in order to provide a basis for identifying the contribution of each to impaired ventilation capacity.

\section{METHODS}

The subjects employed were all healthy young adults, either medical students, or hospital nurses, and were among those described in a previous report (1).

The voluntary ventilation capacity was determined by the standardized procedure previously described (1), using the Benedict-Roth basal metabolism spirometer with soda-lime container and valves removed. The same instrument was used for the vital capacity determination, again with the $\mathrm{CO}_{2}$ absorber removed so that the full volume of expired air remained in the instrument for measurement. Both ventilation capacities and vital capacities were converted to BTPS, using the formula previously reported (1) instead of the basal metabolism tables which are applicable only when the soda-lime is present.

In five normal subjects the vital capacity was experimentally reduced by means of a canvas vest which could be progressively tightened to restrict the respiratory excursion of the chest. In eight normal subjects, resistance to air flow was experimentally increased by inserting plastic tubes into the metal endpiece of the conducting tubes of the spirometer. The plastic tubes were $1 \mathrm{~cm}$. long with various internal diameters.

It was necessary to devise a new technique for determining the maximum expiratory pressure. Previous methods have utilized a mercury manometer. In order to reduce the enormous inertia error, the subject is usually required to maintain the pressure until the mercury comes to rest. This introduces two new difficulties, however; the unpleasant effects of the prolonged Valsalva maneuver conduce to less than maximum effort, and the subject may attempt to maintain the pressure with cheeks and closed glottis instead of the chest. All these difficulties can be avoided by utilizing an inertia-free manometer, such as the aneroid sphygmomanometer. With this particular type of manometer, however, inspiratory pressures cannot be measured.

The maximum expiratory pressure was determined by directing the subject first to sit erect on the edge of a chair, second to take the deepest possible breath, and third to blow as forcefully as possible into the mouthpiece attached by a short length of pressure tubing to the aneroid manometer. The pressure was then read to the nearest $5 \mathrm{~mm}$. of $\mathrm{Hg}$ by the operator. The subject was not required to maintain the pressure for more than the instant required for reading the manometer. It is important that the initial breath be maximal, since the 
pressure that can be developed diminishes with the depth of the initial breath (2). The rate at which the pressure is developed, however, is not critical unless extremely rapid as in a cough, or very slow so that discomfort or fatigue occurs before the peak pressure is attained. After a few trials the subject readily learns to bear down with maximum effect.

\section{Vital capacity}

\section{RESULTS}

As shown in Table I the vital capacity of 194 healthy male subjects averaged $5.13 \pm 0.66$ liters and in 40 healthy female subjects $3.58 \pm 0.53$ liters. These mean values are higher than many that have been reported, but care has not always been taken with respect to the selection of healthy subjects, to proper correction of values to BTPS, and to removal of the $\mathrm{CO}_{2}$ absorber from the spirometer. Reliability coefficients based on duplicate determinations made either at a single sitting or a week apart were found to range from 0.97 to 0.98 .

The voluntary ventilation capacity was also determined in each of the above subjects, yielding the values included in Table I. The correlation coefficient between these two types of capacity determinations was 0.42 for the 194 male subjects and 0.54 for the 40 female subjects. Both coefficients are statistically significant, but of such low order as to demonstrate convincingly that other factors, presumably muscular force and pulmonary resistance, are more important in determining the ventilation capacity than is the vital capacity.

It is of importance to establish the nature of the relationship between ventilation and vital capacities. If, when the other factors remain constant, the relationship is a simple direct proportion, the ratio of the two capacities will remain constant. Under these conditions a capacity ratio may be calculated by dividing the ventilation capacity by the vital capacity and the resulting figure will represent the ventilation capacity per liter of vital capacity. Abnormalities in this capacity ratio would then indicate deviation from normal in either muscular force or air-flow resistance.

In the healthy population sampled, presumably the force and resistance factors (except insofar as they are determined by vital capacity) are randomly and therefore uniformly distributed. Hence, a first approximation to the underlying relationship between the two capacities may be made by examination of these data. Capacity ratios as defined above have been calculated for each of the healthy subjects. As shown in Table I, the mean value of 32.8 is the same for both men and women, implying that the ventilation capacity and vital capacity are reduced proportionately in woman as compared with men. On the average, therefore, 1 liter of vital capacity is capable of

TABLE I

Relationship between vital capacity and voluntary ventilation capacity

\begin{tabular}{|c|c|c|c|c|c|}
\hline & \multicolumn{3}{|c|}{ Normal population } & \multirow{2}{*}{$\begin{array}{l}\text { Vest } \\
\text { exper. }\end{array}$} & \multirow{2}{*}{ Total } \\
\hline & Male & Female & Combined & & \\
\hline 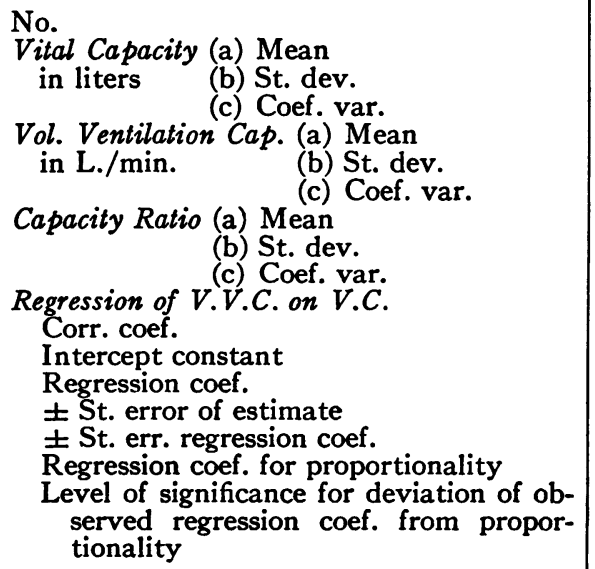 & $\begin{array}{l}194 \\
5.13 \\
0.66 \\
12.8 \\
166.8 \\
20.5 \\
12.3 \\
32.8 \\
4.43 \\
13.5 \\
0.419 \\
99.6 \\
13.1 \\
18.7 \\
2.05 \\
32.5 \\
<1 \%\end{array}$ & $\begin{array}{c}40 \\
3.58 \\
0.53 \\
14.7 \\
115.8 \\
20.9 \\
18.0 \\
32.7 \\
5.34 \\
16.3 \\
\\
0.544 \\
46.7 \\
19.3 \\
17.8 \\
4.83 \\
32.4 \\
2 \%\end{array}$ & $\begin{array}{c}234 \\
4.87 \\
0.89 \\
18.4 \\
158.1 \\
28.2 \\
17.8 \\
32.8 \\
4.58 \\
14.0 \\
0.696 \\
48.5 \\
22.5 \\
20.2 \\
1.52 \\
32.5 \\
<1 \%\end{array}$ & $\begin{array}{c}20 \\
4.54 \\
1.12 \\
24.7 \\
149.9 \\
36.7 \\
24.5 \\
33.3 \\
4.50 \\
13.9 \\
0.866 \\
21.3 \\
28.4 \\
18.9 \\
3.86 \\
33.0 \\
25 \%\end{array}$ & $\begin{array}{c}254 \\
4.84 \\
0.89 \\
18.4 \\
157.4 \\
28.9 \\
18.4 \\
32.8 \\
4.58 \\
14.0 \\
\\
0.719 \\
45.1 \\
23.2 \\
20.1 \\
1.01 \\
32.5 \\
<1 \%\end{array}$ \\
\hline
\end{tabular}


supporting a ventilation capacity of $32.8 \mathrm{~L} . / \mathrm{min}$. in both sexes. It should also be noted that the coefficient of variation is \pm 13.5 per cent, nearly identical with those for vital and ventilation capacities. This, of course, is a reflection of the correlation between the two capacities.

Linear regression equations were fitted to the data on vital and ventilation capacities. As shown in Table $I$, the intercept values for the regression equations are both positive, in contrast to what is required of a direct proportion, and in spite of the obvious fact that the true relationship between these particular variables must pass through the origin. This discrepancy may be only apparent because not statistically significant, or it may be real and indicate a relationship other than linear.

Whether the positive intercepts represent significant deviations from direct proportionality was investigated in the following way, illustrated by the male data. If the regression is a direct proportion, the line must pass through the origin as well as the point representing the mean ventilation capacity (166.8) and the mean vital capacity (5.13). These points define a slope of 32.5 . The observed slope was only 13.1 , however, with a standard error of \pm 2.05 . Using the t-test, this discrepancy is highly significant, implying that the observed slope is not that required for proportionality. Similar results were obtained upon analysis of the female data and those for both sexes combined. It is apparent, however, that as the data improve (higher correlation in the females and extended range in combining the sexes) the regression line more closely approaches a simple proportion (smaller intercept constant and larger regression coefficient). Furthermore, in these experiments, the vital capacity, which is the independent variable, is not measured without error and this biases both the regression coefficient and its standard error downward, thereby favoring the appearance of nonproportionality.

In order to provide a different approach to this problem, the vital capacities of five healthy subjects were experimentally reduced by progressive interference with chest excursion by a canvas vest. Both vital and ventilation capacities were determined under normal conditions and at three levels of restriction by the vest. In this way a range of vital capacity equal to that of the combined sexes was covered without altering muscular force or air-flow resistance (except insofar as these are affected by vital capacity). The results of these experiments are included in Table I. The correlation coefficient is higher (0.87) and the regression equation does not deviate significantly from a direct proportion.

In Figure 1 all three sets of data are plotted. Of the three smooth curves included in the graph, one represents a direct proportion with a constant capacity ratio of 32.5 , another the linear regression line fitted to all the data, and the curved dotted line a statistically fitted parabola passing through the origin. The parabola is probably the best fitting line, but its deviation from the direct proportion, especially in the important range of low vital capacities, is rather slight. Accordingly, for practical purposes it is concluded that in the absence of abnormal disturbances of muscular force and pulmonary resistance, the capacity ratio lies within the normal range, as established above, and that abnormalities in this ratio may be considered to betray the presence of abnormalities in these other factors.

\section{Maximum expiratory pressure}

In 54 male subjects the maximum expiratory pressure was measured for five consecutive trials within a period of a few minutes; in 34 of these subjects three more trials were made approxi-

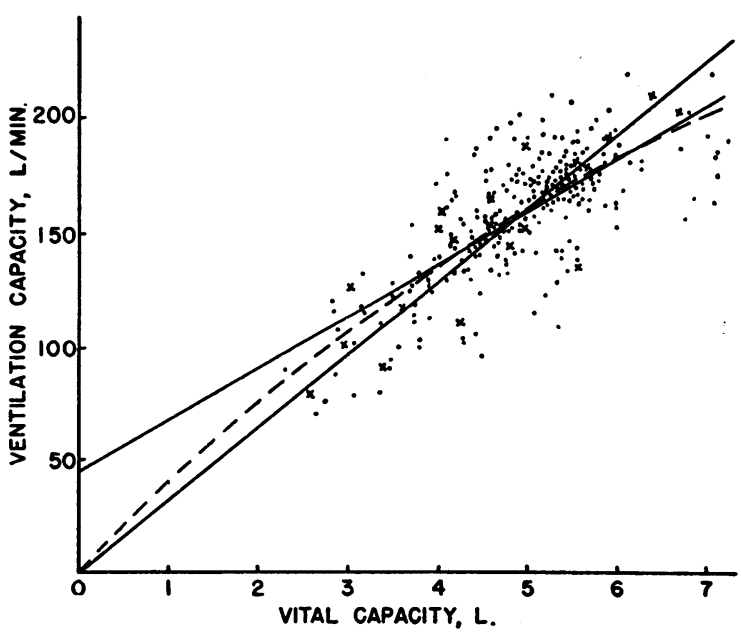

Fig. 1. Ventilation Capacity as a Function of Vital Capacity

Male and female subjects represented by dots, and vest experiments by crosses. 
TABLE II

The maximum expiratory pressure and its correlations

\begin{tabular}{|c|c|c|c|c|c|c|c|}
\hline & & \multicolumn{3}{|c|}{34 Medical students } & \multicolumn{3}{|c|}{54 Medical students } \\
\hline & & Mean & St. dev. & C. var. & Mean & St. dev. & C. var. \\
\hline$M E P$ First Session, & $\begin{array}{l}\text { Trial No. } 1 \\
\text { Trial No. } 2 \\
\text { Trial No. } 3 \\
\text { Trial No. } 4 \\
\text { Trial No. } 5 \\
\text { Mean of Nos. } 4 \text { and } 5 \\
\text { Trial No. } 6 \\
\text { Trail No. } 7 \\
\text { Trial No. } 8 \\
\text { Mean of Nos. } 7 \text { and } 8 \\
\text { ean of two trials } \\
\text { Mean of two trials }\end{array}$ & $\begin{array}{l}120.15 \\
127.35 \\
133.82 \\
141.32 \\
141.62 \\
141.44 \\
141.76 \\
145.44 \\
147.94 \\
146.82 \\
=\end{array}$ & $\begin{array}{l}\overline{-} \\
\overline{-} \\
31.9 \\
33.7 \\
32.3 \\
\overline{37.6} \\
39.3 \\
38.2 \\
=\end{array}$ & $\begin{array}{l}\bar{Z} \\
\bar{Z} \\
22.6 \\
23.8 \\
22.8 \\
\overline{25.9} \\
26.6 \\
26.0 \\
=\end{array}$ & $\begin{array}{l}117.96 \\
125.37 \\
131.85 \\
141.11 \\
139.91 \\
140.80 \\
= \\
= \\
\overline{5.02} \\
165.0\end{array}$ & $\begin{array}{c}\bar{z} \\
\overline{\bar{n}} \\
\pm 32.7 \\
\pm 32.7 \\
32.1 \\
\bar{z} \\
\overline{-} \\
\overline{0.533} \\
25.1\end{array}$ & $\begin{array}{l}\overline{-} \\
\overline{23.2} \\
23.4 \\
22.8 \\
= \\
\overline{-} \\
10.6 \\
15.2\end{array}$ \\
\hline \multicolumn{2}{|c|}{$\begin{array}{l}\text { V.C. First Session, Mean of two trials } \\
\text { V.V.C. First Session, Mean of two trials } \\
\text { Reliability Coefficients } \\
M E P \text { First Session, No. } 4 \text { vs. No. } 5 \\
\text { Second Session, No. } 7 \text { vs. No. } 8 \\
\text { Both Sessions, Nos. } 4 \text { and } 5 \text { vs. } 7 \text { and } 8\end{array}$} & & $\begin{array}{l}0.939 \\
0.968 \\
0.740\end{array}$ & & & $\begin{array}{l}0.225 \\
0.106\end{array}$ & \\
\hline
\end{tabular}

mately one week later. The mean values (Table II) for the first four trials showed progressive improvement from 120 to $141 \mathrm{~mm}$. of $\mathrm{Hg}$ with no further change on the fifth trial. One week later a sixth trial gave nearly an identical initial value, but the seventh and eighth showed a slight further gain to $148 \mathrm{~mm}$. of $\mathrm{Hg}$. The learning factor in this test, therefore, is appreciable, in contrast to the absence of learning in the vital capacity test, and the slight learning of the ventilation capacity test. On the basis of these findings, values for the fourth and fifth trials were averaged to yield the maximum expiratory pressure for each subject. For the 54 subjects, therefore, the mean maximum expiratory pressure was found to be $140.8 \mathrm{~mm}$. of $\mathrm{Hg}$ with a standard deviation of 32.1 and a coefficient of variation of 23 per cent.

The present mean of 140.8 is considerably higher than previous norms based upon a mercury manometer technique. Rahn and his associates (2) reported a mean of $115 \mathrm{~mm}$. of $\mathrm{Hg}$ and have summarized earlier work. The essential difference is attributable to the use of a comparatively inertia-free aneroid manometer.

Reliability coefficients based on duplicate determinations made at a single sitting were quite high, ranging from 0.91 to 0.97 , but fell to 0.74 when based on determinations made a week apart (Table II).
Both vital capacity and ventilation capacity determinations were made on the 54 subjects, but neither correlated significantly with the maximum expiratory pressure. Even the partial correlation between ventilation capacity and expiratory pressure was no better than the zero order correlation, and the capacity ratio likewise failed to correlate with expiratory pressure. Although this complete lack of correlation was not anticipated there are many reasons for expecting a very poor one in these experiments. First, since only healthy subjects were studied no abnormal deviations in either ventilation capacity or respiratory pressure were present. Second, although the inspiratory pressure is presumably as important as the expiratory, it was not measured because of the greater difficulties involved. Most important, however, is the fact that under the dynamic conditions of maximum ventilation only a small and variable portion of the total muscular force remains available for the actual movement of air; the greater portion must be dissipated in overcoming inertia, elasticity and internal friction of the lung and thorax. The maximum expiratory pressure, on the other hand, is measured under static conditions where the entire muscular force can be devoted to the production of intrapulmonic pressure. Although no effect of expiratory pressure on ventilation capacity could be demonstrated in these normal subjects, it 


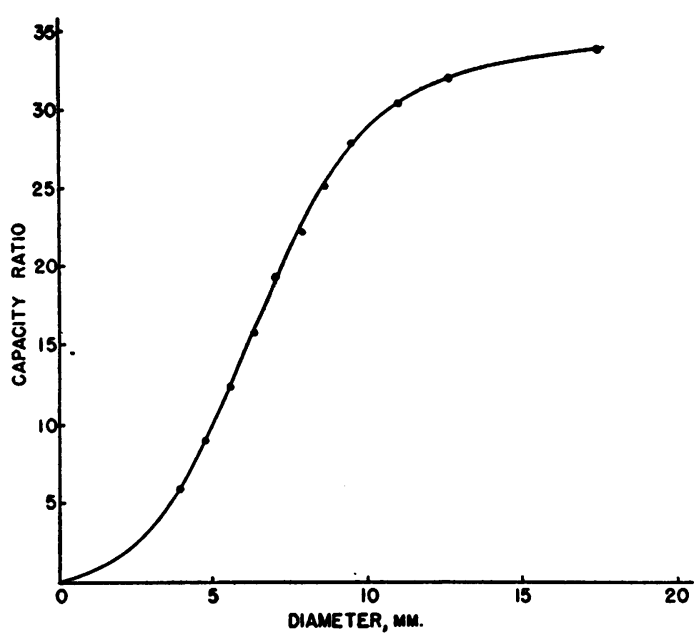

Fig. 2. Ventilation Capacity as a Function of the Diameter of Artificial Resistance Tubes

is inevitable that in severe pathological conditions, such as muscular paralysis, a reduction in expiratory pressure must be accompanied by an impairment of ventilation capacity.

\section{Air-flow resistance}

There is at present no simple method available for measuring the resistance to air flow offered by the respiratory passages of human subjects. In order to demonstrate the effect of this factor, therefore, it became necessary to introduce artificial air-flow resistances in the spirometer system. Plastic tubes $1 \mathrm{~cm}$. long and with various internal diameters were used as resistances. The results obtained in eight healthy subjects are presented in Figure 2. Without added resistance, the ventilation capacity, vital capacity, and capacity ratios averaged 186, 5.55, and 33.7 respectively. As greater resistances were introduced the ventilation capacity was affected gradually at first and then progressively more rapidly. With the greatest resistance the mean ventilation capacity was $33 \mathrm{~L} . / \mathrm{min}$. and the capacity ratio 6.0 ; the vital capacity, however, remained unchanged.

It is apparent from these results that resistance to air flow exerts a profound effect on the ventilation capacity, without influencing the vital capacity, and that this effect is reflected in the capacity ratio.

\section{DISCUSSION}

Determinations of the vital capacity and maximum expiratory pressure, both of which are simple and reliable tests, should greatly facilitate the clinical analysis of causes of impaired ventilation capacity. In general a reduced ventilation capacity may be due to a pulmonary bellows which is either too small, offers too much resistance, or is inadequately powered, or a combination of these. The size can be readily measured by the vital capacity and its effect alone on ventilation capacity can be estimated from the capacity ratio. A reduction in vital capacity without reduction in the capacity ratio implies that only the vital capacity is at fault. On the other hand, a reduction, in the capacity ratio, whether accompanied or not by a change in vital capacity, is evidence of excessive resistance or impaired motive power. The latter can be measured by the maximum expiratory pressure, although there is as yet no basis for estimating its exact contribution to the ventilation capacity. However, the present results indicate that its effects are small unless there is gross muscular impairment. The resistance factor includes both air-flow resistance in the respiratory passage and resistance of the lungs and thorax to deformation due to inertia, rigidity, and internal friction. The former component has been demonstrated to be a powerful factor capable of overwhelming all others; the latter component is probably less important but may be expected to operate especially in obesity and in disease of the bony thorax.

The present scheme emphasizes a more rational application of the much-abused vital capacity test. Over the past century repeated attempts have been made to establish this test as a measure of overall pulmonary function, or of ventilation capacity (3-5). Actually the vital capacity is merely the difference between two static lung volumes and involves no time element. By contrast, the ventilation capacity is a dynamic rate measurement. The attempt to measure one by determining the other involves the wholly unreasonable assumption that the size of the pulmonary bellows exclusively determines its maximum rate of ventilation. The present experiments demonstrate conclusively that air-flow resistance can profoundly impair ventilation capacity without affecting the 
vital capacity at all. It should be clear, therefore, that the vital capacity is not an alternative measure of ventilation capacity, but instead may be considered to be only one of several factors which influence the latter.

From the standpoint of fluid mechanics there are two principal factors (aside from the physical properties of the respired gases) which determine the rate of flow of air in and out of the lungs. The first is the alveolar pressure gradient which actually moves the air, and the second is the geometric resistance offered by the respiratory passages. The former depends upon the product of the total muscular force available and the fraction which can be effectively devoted to the development of air pressure gradients. The latter depends upon the number, the diameter, and general geometry of the respiratory passages. In this scheme the vital capacity does not appear per se as a factor. Its effect, therefore, must operate through either the effective pressure or geometric resistance. For example, a small vital capacity may reflect fewer or smaller conducting passages, or less effective muscular force. Although vital capacity may to some extent affect the pressure and resistance factors, the latter can also vary independently of vital capacity. Only such independent variations in force and resistance are revealed by the capacity ratio.

\section{SUM MARY AND CONCLUSIONS}

1. Procedures, reliability of measurement, and normal values (based on healthy young adults) are reported for determination of the vital capacity, the maximum expiratory pressure, and the capacity ratio, defined as the ratio of voluntary ventilation capacity to vital capacity.
2. The voluntary ventilation capacity was found to correlate weakly $(0.4$ to 0.7$)$ with the vital capacity and not at all (0.10) with the maximum expiratory pressure in normal subjects.

3. Experimental reduction in vital capacity reduced the ventilation capacity proportionately, leaving the ventilation capacity per liter of vital capacity (the capacity ratio) unchanged.

4. The introduction of artificial air-flow resistances profoundly depressed the ventilation capacity and the capacity ratio, without altering vital capacity.

5. A simple scheme is presented for analyzing the causes of reduced ventilation capacity in terms of the size of the pulmonary bellows (vital capacity), the muscular force available for its operation (maximum expiratory pressure), and its resistance to motion and air flow.

\section{BIBLIOGRAPHY}

1. Gray, J. S., Barnum, D. R., Matheson, H. W., and Spies, S. N., Ventilatory function tests. I. Voluntary ventilation capacity. J. Clin. Invest., 1950, $29,677$.

2. Rahn, H., Otis, A. B., Chadwick, L. E., and Fenn, W. O., The pressure-volume diagram of the thorax and lung. Am. J. Physiol., 1946, 146, 161.

3. Sturgis, C. C., Peabody, F. W., Hall, F. C., and Fremont-Smith, F., Clinical studies on respiration: relation of dyspnea to the maximum minute-volume of pulmonary ventilation. Arch. Int. Med., 1922, $29,236$.

4. Peabody, F. W., and Wentworth, J. A., Clinical studies on respiration: the vital capacity of the lungs and its relation to dyspnea. Arch. Int. Med., 1917, 20, 443.

5. Kaltreider, N. L., and McCann, W. S., Respiratory response during exercise in pulmonary fibrosis and emphysema. J. Clin. Invest., 1937, 16, 23. 